\title{
Metal artifact reduction in patients with dental implants using multispectral three-dimensional data acquisition for hybrid PET/MRI
}

Jeanne M Gunzinger ${ }^{1}$, Gaspar Delso ${ }^{2}$, Andreas Boss ${ }^{3}$, Miguel Porto $^{1}$, Helen Davison ${ }^{1}$, Gustav K von Schulthess ${ }^{1}$, Martin Huellner ${ }^{1,4}$, Paul Stolzmann ${ }^{1,4}$, Patrick Veit-Haibach ${ }^{1,3}$ and Irene A Burger ${ }^{1,3^{*}}$

\author{
* Correspondence: \\ irene.burger@usz.ch \\ 'Department of Medical Radiology, \\ Division of Nuclear Medicine, \\ University Hospital Zurich, Ramistr. \\ 100, CH-8091 Zurich, Switzerland \\ ${ }^{3}$ Department of Medical Radiology, \\ Institute of Diagnostic and \\ Interventional Radiology, University \\ Hospital Zurich, Ramistr. 100, \\ $\mathrm{CH}-8091$ Zurich, Switzerland \\ Full list of author information is \\ available at the end of the article
}

\begin{abstract}
Background: Hybrid positron emission tomography/magnetic resonance imaging (PET/MRI) shows high potential for patients with oropharyngeal cancer. Dental implants can cause substantial artifacts in the oral cavity impairing diagnostic accuracy. Therefore, we evaluated new MRI sequences with multi-acquisition variable-resonance image combination (MAVRIC SL) in comparison to conventional high-bandwidth techniques and in a second step showed the effect of artifact size on MRI-based attenuation correction (AC) with a simulation study.

Methods: Twenty-five patients with dental implants prospectively underwent a trimodality PET/CT/MRI examination after informed consent was obtained under the approval of the local ethics committee. A conventional 3D gradient-echo sequence (LAVA-Flex) commonly used for MRI-based AC of PET (acquisition time of $14 \mathrm{~s}$ ), a T1w fast spin-echo sequence with high bandwidth (acquisition time of $3.2 \mathrm{~min}$ ), as well as MAVRIC SL sequence without and with increased phase acceleration (MAVRIC, acquisition time of 6 min; MAVRIC-fast, acquisition time of $3.5 \mathrm{~min}$ ) were applied. The absolute and relative reduction of the signal void artifact was calculated for each implant and tested for statistical significance using the Wilcoxon signed-rank test. The effect of artifact size on PET AC was simulated in one case with a large tumor in the oral cavity. The relative difference of the maximum standardized uptake value $\left(S U V_{\text {max }}\right)$ in the tumor was calculated for increasing artifact sizes centered over the second molar.
\end{abstract}

Results: The absolute reduction of signal void from LAVA-Flex sequences to the T1-weighted fast spin-echo (FSE) sequences was $416 \mathrm{~mm}^{2}$ (range 4 to 2,010 $\mathrm{mm}^{2}$ ) to MAVRIC $481 \mathrm{~mm}^{2}$ (range 12 to 2,288 $\mathrm{mm}^{2}$ ) and to MAVRIC-fast $486 \mathrm{~mm}^{2}$ (range 39 to $2,209 \mathrm{~mm}^{2}$ ). The relative reduction in signal void was significantly improved for both MAVRIC and MAVRIC-fast compared to T1 FSE (-75\%/-78\% vs. $-62 \%, p<0.001$ for both). The relative error for SUV $\max$ was negligible for artifacts of $0.5-\mathrm{cm}$ diameter $(-0.1 \%)$, but substantial for artifacts of $5.2-\mathrm{cm}$ diameter $(-33 \%)$.

Conclusions: MAVRIC-fast could become useful for artifact reduction in PET/MR for patients with dental implants. This might improve diagnostic accuracy especially for patients with tumors in the oropharynx and substantially improve accuracy of PET quantification.

Keywords: MAVRIC; Attenuation correction; Signal voids; Image noise

\section{Springer}

(c) 2014 Gunzinger et al; licensee Springer. This is an Open Access article distributed under the terms of the Creative Commons Attribution License (http://creativecommons.org/licenses/by/2.0), which permits unrestricted use, distribution, and reproduction in any medium, provided the original work is properly credited. 


\section{Background}

In head and neck tumor staging, computed tomography (CT) and magnetic resonance imaging (MRI) play an important role in the evaluation of local tumor extension, since clinical and endoscopic examination often results in underestimation of disease, as deep infiltration of the surrounding tissues can be hard to detect [1-3]. Generally, diagnostic imaging is performed after clinical and endoscopic examination for staging and therapy planning and as a base for further follow-up examinations [4]. Functional imaging like fluorodeoxyglucose (FDG) positron emission tomography (PET) measures the metabolic activity and is superior in nodal staging compared to CT or MRI [5,6]. For accurate anatomic localization and spatial resolution, cross-sectional hybrid imaging methods like PET/CT are superior than PET alone [7,8]. For oropharyngeal cancer, T-staging could be optimized with PET/MRI compared to PET/CT, due to a higher soft tissue contrast $[9,10]$. This raises the interest to improve PET/MRI protocols for specific indications taking into account organ and pathology dependent adaptations $[11,12]$. PET/MRI has already been shown to be feasible for imaging head and neck cancer with a whole-body PET/MRI system without impairment of PET quality [13].

The two main problems for MRI of the oral cavity are patient motion and artifacts of dental alloys due to magnetic susceptibility artifacts [14]. To reduce patient motion, a short acquisition time is favorable and the patient should be well instructed and have a comfortable position [14]. The extent of artifacts from dental alloys depends on the composition, with ferromagnetic material causing strongest artifacts [15]. However, even titanium alloys generally considered 'MRI-compatible' may lead to significant susceptibility artifacts due to their paramagnetic properties [16]. Different MRI sequences are differently prone to those susceptibility artifacts depending on the spin excitation technique, data acquisition strategy, and receiver bandwidth [17-20]. Artifacts might appear as signal voids, hyperintense signals caused by signal pile-up due to distortion of spatial encoding, or geometric distortions [15,18,21]. An optimized MRI sequence design can reduce these artifacts significantly [14] and thereby improve diagnostic accuracy and also reduce artifacts for MR-based attenuation correction (AC), since large signal voids can lead to substantial underestimation of FDG uptake within the area of the artifact when MRI-based AC is performed [22].

Conventional strategies to optimize the image quality close to metal implants are a high bandwidth per voxel, 3D spatial encoding, a high-resolution matrix, and a multiecho spin-echo (SE) sequence or turbo/fast SE sequence [23].

The relatively new multi-acquisition variable-resonance image combination (MAVRIC) as well as the slice encoding for metal artifact correction (SEMAC) technique has shown very promising results in reducing susceptibility artifacts in arthroplasty imaging [24-27]. MAVRIC images can be used in extreme off-resonance conditions by splitting very large spectral distributions into independently imaged frequency bins with a multispectral three-dimensional technique-space composition [28]. SEMAC uses a slice selection gradient for excitation and a view-angle tilting (VAT) compensation gradient for readout [24]. MAVRIC and SEMAC showed significantly smaller artifact extent compared to fast spin-echo (FSE) imaging [24].

Given the good results of MAVRIC in arthroplasty imaging, we investigated this technique for its capability to depict the oral cavity in the presence of metallic dental implants by comparing artifacts in MRI datasets acquired with FSE, standard MAVRIC 
SL, and a MAVRIC-fast with an increased phase acceleration allowing a shorter repetition time (TR), resulting in notably shorter acquisition time. Furthermore, a simulation study was performed to calculate the effect of different artifact sizes on maximum standardized uptake value $\mathrm{SUV}_{\max }$ in PET images after MRI-based AC.

\section{Methods}

This prospective study was conducted with patients referred for FDG PET/CT who gave written informed consent for additional MRI scans during the FDG uptake time. Patients were included if they had dental implants and did not have any contraindication for MRI. Between September 2013 and January 2014, 25 patients (19 males and 6 females) were included. The study was carried out with the approval of the local ethics committee. Examinations were performed using a sequential trimodality PET/CT-MRI system consisting of a GE Healthcare Discovery 750w 3T MRI and a GE Healthcare Discovery 690 PET/CT (GE Healthcare, Milwaukee, WI, USA) [10]. A shuttle device enabling to transfer the patient from the MRI to the PET/CT without changing the patient's position was used. Standard PET/CT was acquired and axial images of the oral cavity were obtained from CT $(120 \mathrm{kV}$, tube current with automated dose modulation with 60 to $440 \mathrm{~mA} /$ slice).

The in-phase images of a dual-echo gradient-echo pulse sequence (LAVA-Flex (GE Healthcare, Milwaukee, WI, USA) with TR $4.3 \mathrm{~ms}$, echo time (TE) $1.3 \mathrm{~ms}$, a matrix size of $288 \times 224$ with a spatial resolution of $1.7 \times 2.2 \times 4.0 \mathrm{~mm}$; covering a field of view of $50 \mathrm{~cm}$, using a bandwidth of $142.86 \mathrm{kHz}$, with an acceleration factor of 2 and a total acquisition time of $14 \mathrm{~s}$ ) commonly used in whole-body MR imaging for AC of PET images were used as a reference [29,30]. A 2D encoded T1-weighted FSE sequence with increased bandwidth (TR $339 \mathrm{~ms}$, TE $13.6 \mathrm{~ms}$, slice thickness $3 \mathrm{~mm}$, receiver bandwidth $142.86 \mathrm{kHz}$, acceleration factor of 1.75 , acquisition time of $3.16 \mathrm{~min}$ ) was acquired in axial orientation. Additionally, two MAVRIC sequences were applied, with 24 spectral bins of $2.25 \mathrm{kHz}$ each to cover $\pm 11 \mathrm{kHz}$ (MAVRIC SL, GE Healthcare, Milwaukee, WI, USA). The standard MAVRIC SL with a phase acceleration of 2 resulted in a TR of 4,000 ms and a TE of $7.6 \mathrm{~ms}$ (acquisition time of $6 \mathrm{~min}$ ). To reduce scan time, the phase acceleration was increased to 3 for MAVRIC-fast allowing a shorter TR of 3,000 ms (TE 7.6 ms), resulting in an acquisition time of $3.5 \mathrm{~min}$. All three tested sequences had identical matrix sizes of $384 \times 256$ with an in-plane spatial resolution of $0.9 \mathrm{~mm}$.

\section{Quantitative analysis}

The signal void was quantitatively assessed for every implant using a commercially available viewing workstation (GE Advantage Windows 4.4). On the axial images of all four sequences, the largest diameter $a_{1}$ and the corresponding orthogonal diameter $a_{2}$ were measured by a board-certified radiologist [IAB]. The area of the artifact was calculated by assuming the shape of the artifact to be elliptical using the equation $A=\pi \times\left(a_{1} / 2\right) \times\left(a_{2} / 2\right)$, with $A$ meaning the area of the ellipse.

\section{Qualitative analysis}

The qualitative image analysis was performed by two board-certified radiologists [IAB, $\mathrm{PVH}]$. Both compared the four sequences independently and assessed the delineation 
of anatomical details of the oral cavity on a five-point scale with $1=$ good depiction of anatomical structures, $2=$ structures visible with slight blurring, $3=$ oral cavity visible with substantial blurring, $4=$ oral cavity only partially visible, and $5=$ oral cavity not assessable. Furthermore, the image quality was assessed for spatial blurring and image noise on a five-point scale: $1=$ no artifacts, 2 = barely visible artifacts, $3=$ visible artifacts without diagnostic impairment, $4=$ diagnostic impairment, and $5=$ severe artifacts, non-diagnostic [27]. Hyperintense ringing artifacts around dental alloys were noted separately.

Based on the assessment of spatial blurring on LAVA-Flex sequences, two groups were generated: group 1 with low to moderate artifacts (categories 1 to 3 ) and group 2 with blurring artifacts impairing diagnosis (categories 4 and 5). Differences in qualitative data (anatomic distinction, blurring, or image noise) were compared for T1-FSE and MAVRIC-fast between both groups.

\section{MRI-based PET AC}

To estimate the effect of artifact size on PET quantification if MRI sequences are used for $\mathrm{AC}$, we performed a simulation analysis for one patient with a large carcinoma in the right tonsil. Therefore, artifacts of various sizes were artificially inserted into the $\mathrm{AC}$ atlas routinely used for the PET/MR reconstruction. The simulated artifacts were created by inserting a spherical volume into the image and setting the signal to 0 within the volume. The artifacts were all centered over the second molar in the right maxilla and spherical in shape with increasing diameters from 0.5 to $5 \mathrm{~cm}$. The difference between the baseline image, without artifact, and each reconstructed image with an artificial artifact was calculated. The normalized difference between the baseline PET and artifact-corrected PET was used to produce a contour map showing the percentage difference from baseline in each area of the image.

\section{Statistics}

Statistic evaluation was performed with statistical software (SPSS Statistics 22.0, Chicago, IL, USA). The LAVA-Flex sequence was used as a reference. Differences in signal void areas were assessed with the Wilcoxon signed-rank test (KolmogorovSmirnov test: $p<0.05)$. Absolute and relative reduction of artifact sizes were calculated for T1-FSE, MAVRIC SL, and MAVRIC-fast sequences and compared using the Wilcoxon signed-rank test. Differences in scores for the qualitative data (anatomic distinction, blurring, or image noise) were compared using the Wilcoxon signed-rank test. Significance level was set at a $p$ value of $<0.05$. Agreement between the two readers was determined using Cohen's kappa, with $\kappa$ values of 0 indicating poor agreement, 0.01 to 0.2 slight agreement, 0.21 to 0.40 fair agreement, 0.41 to 0.60 moderate agreement, 0.61 to 0.80 good agreement, and 0.81 to 1 excellent agreement [31]. Isocontour maps showing the percentage difference between PET scans after AC with baseline MR images and MR images with increasing artifact size were calculated using MATLAB Software version 2013b (MathWorks Inc., Natick, MA, USA). 


\section{Results}

A total of 46 dental implants could be identified in the 25 patients with an average age of 60 years (range 28 to 76 years) and average weight of $74 \mathrm{~kg}$ (range 44 to $109 \mathrm{~kg}$ ). Image quality and acquisition were acceptable for all patients.

\section{Quantitative assessment}

The largest artifact size of $612 \mathrm{~mm}^{2}$ on axial images was observed on LAVA-Flex sequences and could be reduced to $195 \mathrm{~mm}^{2}$ for T1-FSE sequence to $131 \mathrm{~mm}^{2}$ for MAVRIC SL and to $126 \mathrm{~mm}^{2}$ for MAVRIC-fast (Table 1).

Using LAVA-Flex as a reference, the absolute artifact reduction for T1-FSE was smaller (mean $417 \mathrm{~mm}^{2}$ ) than that for MAVRIC SL with a mean of $481 \mathrm{~mm}^{2}$ or MAVRIC-fast with a mean of $486 \mathrm{~mm}^{2}(p<0.001)$. There was no statistically significant difference between the absolute reduction of MAVRIC SL and MAVRIC-fast $(p=0.064)$ (Table 1, Figure 1a).

The relative artifact reduction with T1-FSE showed a mean of $-62 \%$. Both MAVRIC SL and MAVRIC-fast improved the artifact reduction compared to T1-FSE with a mean of $-75 \%(p<0.001)$ and $-78 \%(p<0.001)$, respectively (Table 1, Figure 1b). MAVRIC-fast improved relative artifact reduction compared to MAVRIC SL significantly $(p=0.017)$.

\section{Qualitative assessment}

The worst image quality for all three criteria was noted for LAVA-Flex images by both readers with a mean of $3.80( \pm 0.71)$ and $3.64( \pm 0.81)$ for anatomic distinction, 3.96 $( \pm 0.74)$ and $4.00( \pm 0.71)$ for blurring, and $3.84( \pm 0.47)$ and $3.68( \pm 0.63)$ for image noise, for readers 1 and 2, respectively (Table 2, Figure 2).

There was an increase in image noise for MAVRIC-fast compared to MAVRIC SL for both readers, reaching statistical significance for reader $2(p=0.011)$. For anatomical distinction or spatial blurring, there was no relevant difference between MAVRIC SL and MAVRIC-fast.

Table 1 Overview of artifact sizes in axial slides from dental alloys in the applied sequences

\begin{tabular}{lcccc}
\hline & Minimum & Maximum & Mean & SD \\
\hline Size of artifact $\left(\mathrm{mm}^{2}\right)$ & & & & \\
LAVA-Flex & 81.7 & $2,711.5$ & 611.7 & 578.2 \\
T1-FSE & 36.3 & $1,169.0$ & 194.9 & 210.6 \\
MAVRIC & 15.2 & 891.6 & 130.8 & 157.3 \\
MAVRIC-fast & 11.9 & 880.6 & 125.9 & 167.8 \\
Absolute reduction of artifact $\left(\mathrm{mm}^{2}\right)^{2}$, compared to LAVA-Flex & & & & \\
T1-FSE & -3.7 & $-2,009.5$ & -416.8 & 417.9 \\
MAVRIC & -11.5 & $-2,287.5$ & -480.9 & 463.6 \\
MAVRIC-fast & -39.2 & $-2,208.8$ & -485.8 & 450.3 \\
Relative reduction of artifact (\%), compared to LAVA-Flex & & & & \\
T1-FSE & -04 & -88 & -62 & 19 \\
MAVRIC & -11 & -96 & -75 & 19 \\
MAVRIC-fast & -37 & -96 & -78 & 17 \\
\hline SD standard deviation. & & & &
\end{tabular}

SD standard deviation. 

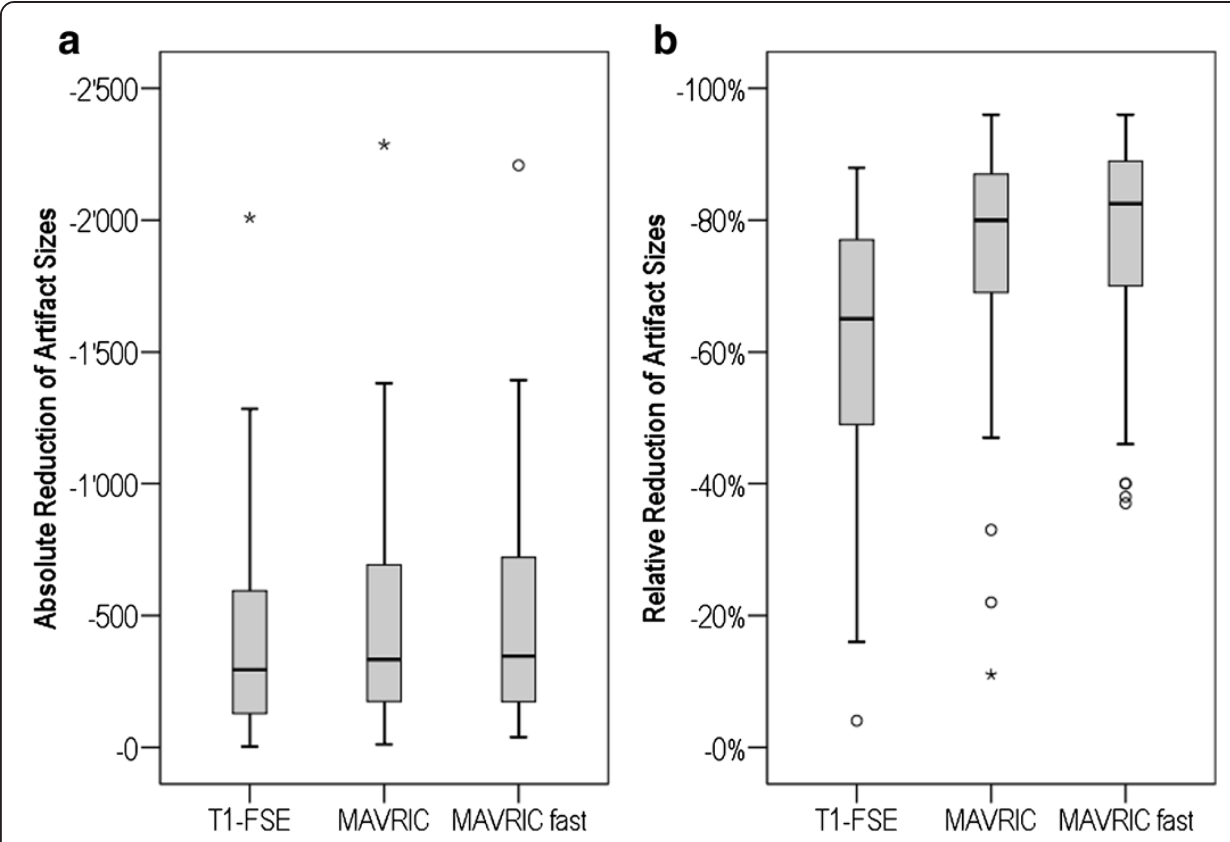

Figure 1 Box plot illustrating the absolute and relative reduction of artifact size in comparison to LAVA-Flex. (a) Box plot illustrating the absolute reduction of artifact size in comparison to LAVA-Flex $\left(\mathrm{mm}^{2}\right)$. All three sequences show a broad spread of reduction, reaching from 4 up to 2,010 $\mathrm{mm}^{2}$ for T1-FSE, 12 up to 2,288 $\mathrm{mm}^{2}$ for MAVRIC, and from 39 up to 2,209 $\mathrm{mm}^{2}$ for MAVRIC-fast (Table 1). (b) Box plot illustrating the relative reduction of artifact size in comparison to LAVA-Flex (\%). The relative artifact reduction was significantly higher for MAVRIC sequences compared to T1-FSE, with a mean of $-62 \%$ for the T1-FSE sequence, $-75 \%$ for MAVRIC, and $-78 \%$ for MAVRIC-fast (Table 1).

Regarding spatial blurring, T1-FSE had substantially more artifacts with a mean of $2.64( \pm 0.76)$ and 2.84 $( \pm 0.80)$ than MAVRIC SL (mean $1.36( \pm 0.64)$ and 1.44 $( \pm 0.65)$, $p<0.001)$ or MAVRIC-fast (mean $1.36( \pm 0.63)$ and $1.40( \pm 0.65), p<0.001)$. Anatomical distinction was overall slightly better for MAVRIC-fast compared to T1-FSE. However, both readers rated image noise significantly better for T1-FSE compared to MAVRIC SL and MAVRIC-fast $(p<0.001)$.

On 23 (92\%) of the images of the LAVA-Flex sequence, the artifacts by the dental alloys showed multiple hyperintense rings (Figure 3). Both readers identified hyperintense ring artifacts on T1-FSE images in 12 cases (48\%), while such an artifact was visible only in one case on MAVRIC SL and MAVRIC-fast sequences.

For anatomical distinction, a moderate to excellent inter-observer agreement was noted ( $\kappa=0.58$ to 0.85 ). For image quality, inter-observer agreement is good to excellent regarding blurring ( $\kappa=0.69$ to 0.93 ) and moderate to excellent for image noise ( $\kappa=0.54$ to 0.89 ) (Table 2).

Subgroup analysis for patients with low to moderate (1 to 3 ) blurring artifacts on LAVA-Flex sequence (group 1) and patients with extensive to non-diagnostic blurring (group 2) was performed. For both readers, there was no significant improvement of spatial blurring with MAVRIC-fast compared to T1-FSE $(p=0.102)$ in group 1. For group 2, MAVRIC-fast led to only barely visible artifacts (mean 1.4 and 1.5, respectively), while T1-FSE showed artifacts impairing diagnostic accuracy in four cases with a mean of 2.8 and 3.0, respectively (Table 3). 
Table 2 Overview of qualitative image analysis and inter-observer agreement

\begin{tabular}{|c|c|c|c|c|}
\hline & Reader 1 & Reader 2 & Kappa (к) & Agreement \\
\hline \multicolumn{5}{|l|}{ LAVA-Flex } \\
\hline Distinction of anatomy & $3.8 \pm 0.7$ & $3.6 \pm 0.8$ & 0.75 & Good \\
\hline Blurring & $4.0 \pm 0.7$ & $4.0 \pm 0.7$ & 0.93 & Excellent \\
\hline Noise & $3.8 \pm 0.5$ & $3.7 \pm 0.6$ & 0.54 & Moderate \\
\hline \multicolumn{5}{|l|}{ T1-FSE } \\
\hline Distinction of anatomy & $1.8 \pm 0.8$ & $2.1 \pm 0.8$ & 0.58 & Moderate \\
\hline Blurring & $2.6 \pm 0.7$ & $2.8 \pm 0.8$ & 0.69 & Good \\
\hline Noise & $1.6 \pm 0.8$ & $1.5 \pm 0.8$ & 0.63 & Good \\
\hline \multicolumn{5}{|l|}{ MAVRIC } \\
\hline Distinction of anatomy & $1.9 \pm 0.6$ & $1.8 \pm 0.6$ & 0.85 & Excellent \\
\hline Blurring & $1.4 \pm 0.6$ & $1.4 \pm 0.6$ & 0.83 & Excellent \\
\hline Noise & $2.1 \pm 0.5$ & $2.0 \pm 0.4$ & 0.89 & Excellent \\
\hline \multicolumn{5}{|l|}{ MAVRIC-fast } \\
\hline Distinction of anatomy & $1.8 \pm 0.4$ & $1.6 \pm 0.5$ & 0.72 & Good \\
\hline Blurring & $1.4 \pm 0.6$ & $1.4 \pm 0.6$ & 0.91 & Excellent \\
\hline Noise & $2.2 \pm 0.5$ & $2.4 \pm 0.6$ & 0.76 & Good \\
\hline
\end{tabular}

Qualitative image assessment was done by two readers using a five-point scale from 1 (good depiction/no artifacts) to 5 (not assessable/non-diagnostic). Data are mean \pm standard deviation. Agreement rating: $k=0$ no agreement; 0.01 to 0.2 slight agreement; 0.21 to 0.40 fair agreement; 0.41 to 0.60 moderate agreement; 0.61 to 0.80 good agreement; and 0.81 to 1 excellent agreement.

\section{Effect of MRI-based PET AC}

The atlas-based MRI AC of the PET data yielded reference PET values in the tumor with a SUV $V_{\max }$ of $25 \mathrm{~g} / \mathrm{ml}$. The tumor was located at the base of the tongue with a size of $2.3 \times 3.7 \times 3 \mathrm{~cm}$. The distance between the hottest voxel within the tumor and the center of the artifact was $5 \mathrm{~mm}$. The absolute and relative change of $\mathrm{SUV}_{\max }$ with increasing diameters of the artificial artifact is given in Table 4. While a signal void of $0.5 \mathrm{~cm}$ did not cause any significant change $(-0.1 \%), 5 \mathrm{~cm}$ led to substantial underestimation of tumor activity of $-33 \%$ in our selected case (Figure 4).

The isocontour maps illustrating the percentage difference from baseline in each area of the image are given for three cases with increasing artifact sizes from 0.5 to $5 \mathrm{~cm}$ (Figure 5).

\section{Discussion}

With this study, we can confirm that MAVRIC is also capable of reducing artifacts from dental implants within the oral cavity. Taking LAVA-Flex images as a reference, the reduction of the artifact size in the axial plane was around $-75 \%$ for MAVRIC SL (and $-78 \%$ for MAVRIC-fast). This is also a significant improvement compared to the artifact reduction accomplished by a T1-FSE sequence with large bandwidth (-62\%). There was a very broad range of signal void sizes for all sequences, due to the various sizes and compositions of the dental alloys [15]. For patients with moderate artifacts in LAVA-Flex (group 1, grades 1 to 3), T1-FSE yielded good image quality with small artifacts due to dental alloys. For patients with extensive blurring due to dental alloys in LAVA-Flex (group 2, grades 4 and 5), spatial blurring in T1-FSE was significantly higher compared to MAVRIC-fast ( $p<0.001$ for both readers) (Table 3). 

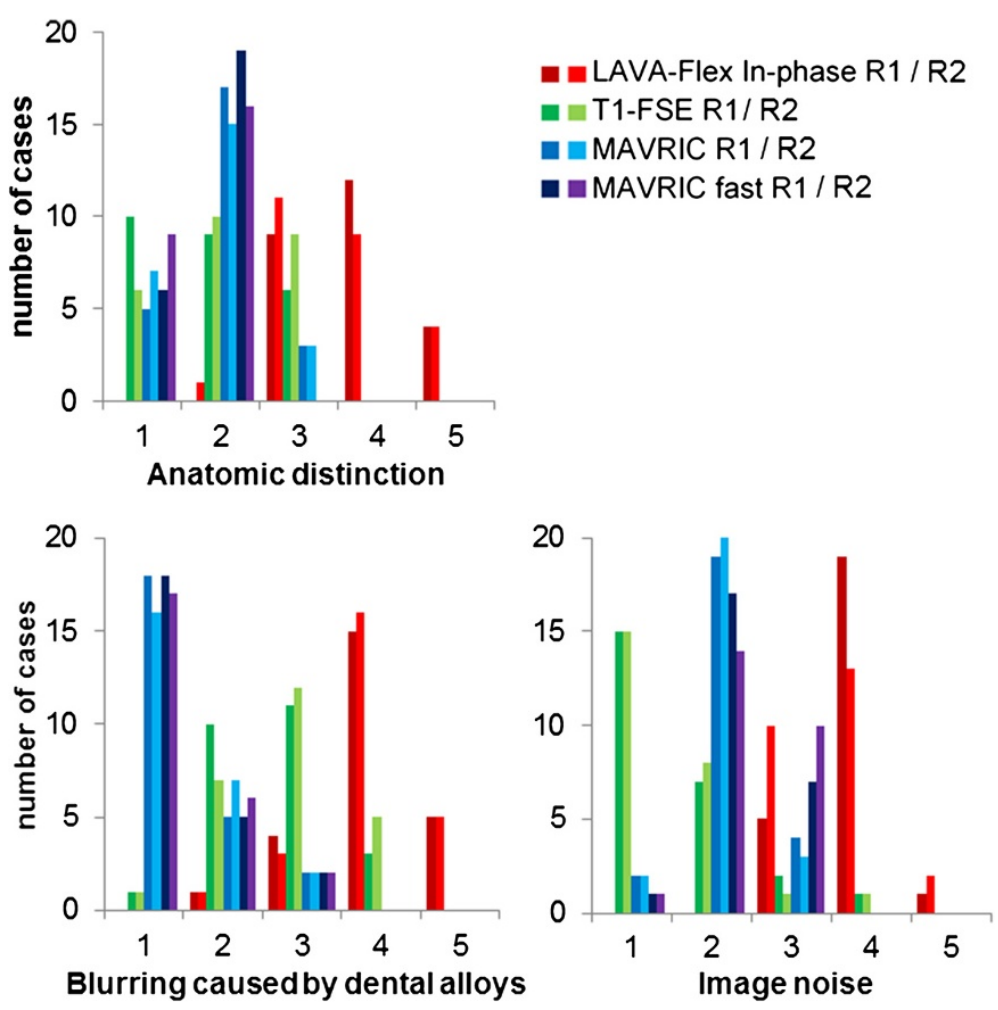

Figure 2 Qualitative analysis of all four sequences for each patient $(n=25)$ by two readers (R1/R2). For anatomic distinction of the floor of the mouth (with $1=$ good depiction of anatomical structures, 2 = structures visible with slight blurring, $3=$ oral cavity visible with substantial blurring, $4=$ oral cavity only partially visible, and $5=$ oral cavity not assessable) as well as blurring caused by dental alloys and image noise assessed on a five-point scale ( $1=$ no artifacts, 2 = barely visible artifacts, $3=$ visible artifacts without diagnostic impairment, 4 = diagnostic impairment, and 5 = severe artifacts, non-diagnostic).

MAVRIC-fast was optimized to reduce the acquisition time of the conventional MAVRIC SL sequence protocol for potential integration into a whole-body PET/MRI protocol. By increasing the phase acceleration from 2 to 3, the echo train length (ETL) was reduced. As susceptibility artifacts increase with echo time [32], this time optimization step also resulted in a further reduction of the size of signal voids. On the other hand, the signal-to-noise ratio decreases with phase acceleration leading to an overall higher image noise for MAVRIC-fast compared to MAVRIC (Table 2). An increase of phase acceleration of MAVRIC, therefore, might only be feasible in areas with a sufficient signal-to-noise ratio obtained by a dedicated receiver coil. In the oral cavity, blurring by dental alloys was approximately equal for MAVRIC SL and MAVRIC-fast, but the MAVRIC-fast technique with TR of 3,000 ms resulted in an acquisition time of $3.5 \mathrm{~min}$, compared to $6 \mathrm{~min}$ for MAVRIC SL. This gives a reasonable scan time for clinical use, comparable with that of the T1-FSE sequence of $3.2 \mathrm{~min}$. Overall, MAVRIC-fast has shown similar imaging results as MAVRIC SL within a favorable acquisition time.

There was only one patient with hyperintense signals due to dental implants distorting MAVRIC SL/MAVRIC-fast images, while T1-FSE images had this artifact in 12 cases $(48 \%)$. Such artifacts can lead to non-interpretable images and misdiagnoses; it is therefore favorable to reduce them as much as possible. Furthermore, MRI data are 


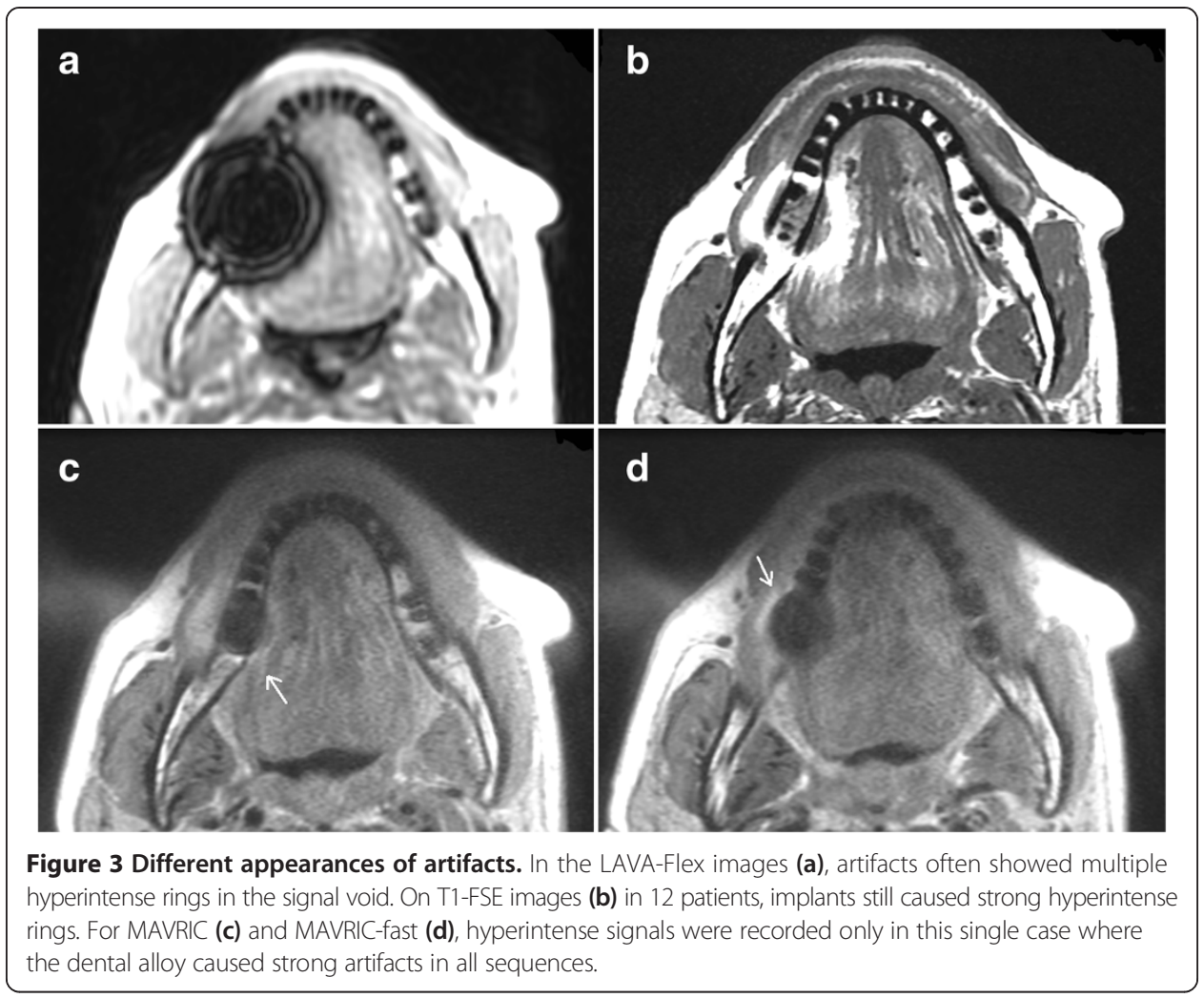

used for AC of PET data in PET/MRI hybrid systems. High image quality without substantial signal voids is favorable for AC [22]. For CT-based AC, it is well known that metal artifacts can lead to false positive findings around prosthesis in PET/CT [33]. In our study, all the obtained CT images showed strong metal artifacts caused by the dental alloys (Figure 6). Therefore, CT-based AC is not a reliable gold standard either. Compared to CT-based AC, the DIXON-based MRI AC (LAVA-Flex) is rather underestimating PET activity in areas of large signal voids [34]. Therefore, large signal voids

Table 3 Image quality of T1-FSE and MAVRIC-fast depending on LAVA-Flex assessment concerning blurring

\begin{tabular}{|c|c|c|c|c|c|c|}
\hline & \multicolumn{3}{|c|}{ Reader 1} & \multicolumn{3}{|c|}{ Reader 2} \\
\hline & T1-FSE & MAVRIC-fast & $p$ value & T1-FSE & MAVRIC-fast & $p$ value \\
\hline \multicolumn{7}{|c|}{ Distinction of anatomy } \\
\hline Group 1 & $1.00 \pm 0.00$ & $1.80 \pm 0.45$ & 0.046 & $1.25 \pm 0.50$ & $1.50 \pm 0.58$ & 0.564 \\
\hline Group 2 & $2.05 \pm 0.76$ & $1.75 \pm 0.44$ & 0.109 & $2.29 \pm 0.72$ & $1.67 \pm 0.48$ & $0.001^{*}$ \\
\hline \multicolumn{7}{|l|}{ Blurring } \\
\hline Group 1 & $2.00 \pm 0.71$ & $1.20 \pm 0.45$ & 0.102 & $2.00 \pm 0.82$ & $1.00 \pm 0.00$ & 0.102 \\
\hline Group 2 & $2.80 \pm 0.70$ & $1.40 \pm 0.68$ & $<0.001^{*}$ & $3.00 \pm 0.71$ & $1.48 \pm 0.68$ & $<0.001^{*}$ \\
\hline \multicolumn{7}{|l|}{ Noise } \\
\hline Group 1 & $1.00 \pm 0.00$ & $2.00 \pm 0.71$ & 0.059 & $1.00 \pm 0.00$ & $2.00 \pm 0.82$ & 0.102 \\
\hline Group 2 & $1.70 \pm 0.87$ & $2.30 \pm 0.47$ & $0.003^{*}$ & $1.62 \pm 0.81$ & $2.43 \pm 0.51$ & $<0.001^{*}$ \\
\hline
\end{tabular}

Image quality was assessed by two readers using a five-point scale from 1 (good depiction/no artifacts) to 5 (not assessable/ non-diagnostic), 3 = without diagnostic impairment and $4=$ with impairment. Data are mean \pm standard deviation. After Bonferroni correction, statistical significance is denoted by $p<0.0167(*=$ statistically significant). Group 1: blurring by dental alloys in LAVA-Flex was rated 1, 2, or 3. Group 2: blurring by dental alloys in LAVA-Flex was rated 4 or 5 . 
Table 4 Change of SUV max $_{\operatorname{mith}}$ win the tumor with increasing sizes of the artificial artifact

\begin{tabular}{lcc}
\hline Artifact diameter $(\mathbf{c m})$ & $\mathbf{S U V}_{\mathbf{m a x}}(\mathbf{g} / \mathbf{m l})$ & Difference in $_{\mathbf{S U V}} \mathbf{m a x}$ \\
\hline 0.0 & 25 & 0.0 \\
0.5 & 25 & -0.1 \\
1.4 & 22.9 & -8.4 \\
2.3 & 21.2 & -15.1 \\
3.3 & 20.1 & -19.5 \\
4.2 & 18.2 & -27.4 \\
5.2 & 16.7 & -33.4 \\
\hline
\end{tabular}

impair PET AC for PET/MRI [35]. The size of signal voids might not translate into identical signal voids on MR attenuation maps; however, a substantial reduction of artifact size will also generate smaller signal voids on MR attenuation maps. The impact of artifact size on PET values could be shown in our simulation study, where artifacts of $19 \mathrm{~cm}^{2}$ lead to an underestimation of $\mathrm{SUV}_{\max }$ of $33 \%$, in a tumor nearby the inserted artifact. In our patient population, artifact size was up to $27 \mathrm{~cm}^{2}$ for LAVA-Flex but only 8.9 and $8.8 \mathrm{~cm}^{2}$ for MAVRIC SL and MAVRIC-fast, respectively. Nevertheless, the presented MRI sequences could not completely reduce artifacts from dental implants
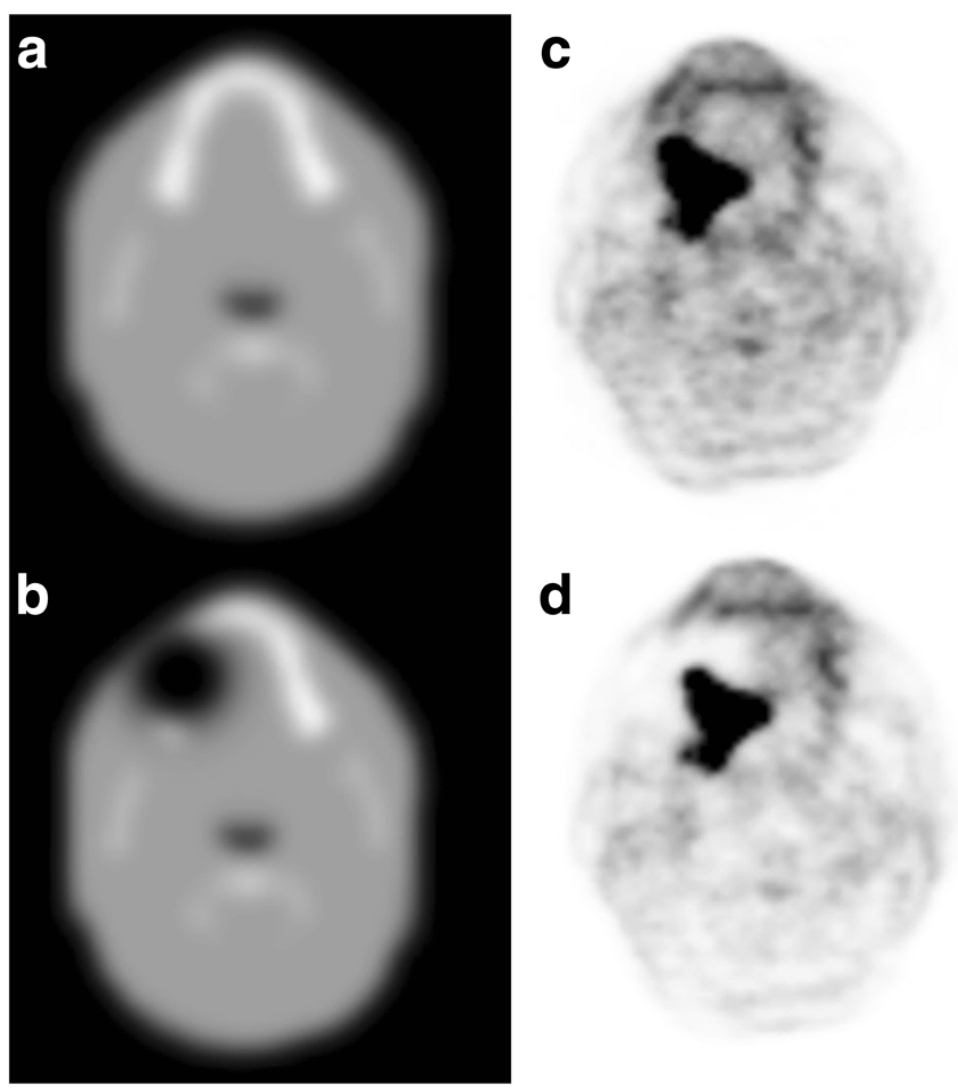

Figure 4 Axial PET images after attenuation correction without and with artifact. (a) Axial image of the used MR atlas image for attenuation correction. (b) The same image with the inserted artifact over the second left molar. (c) Base line axial PET image after attenuation correction using the original MR atlas. (d) Corresponding axial PET image after attenuation correction with signal void. 


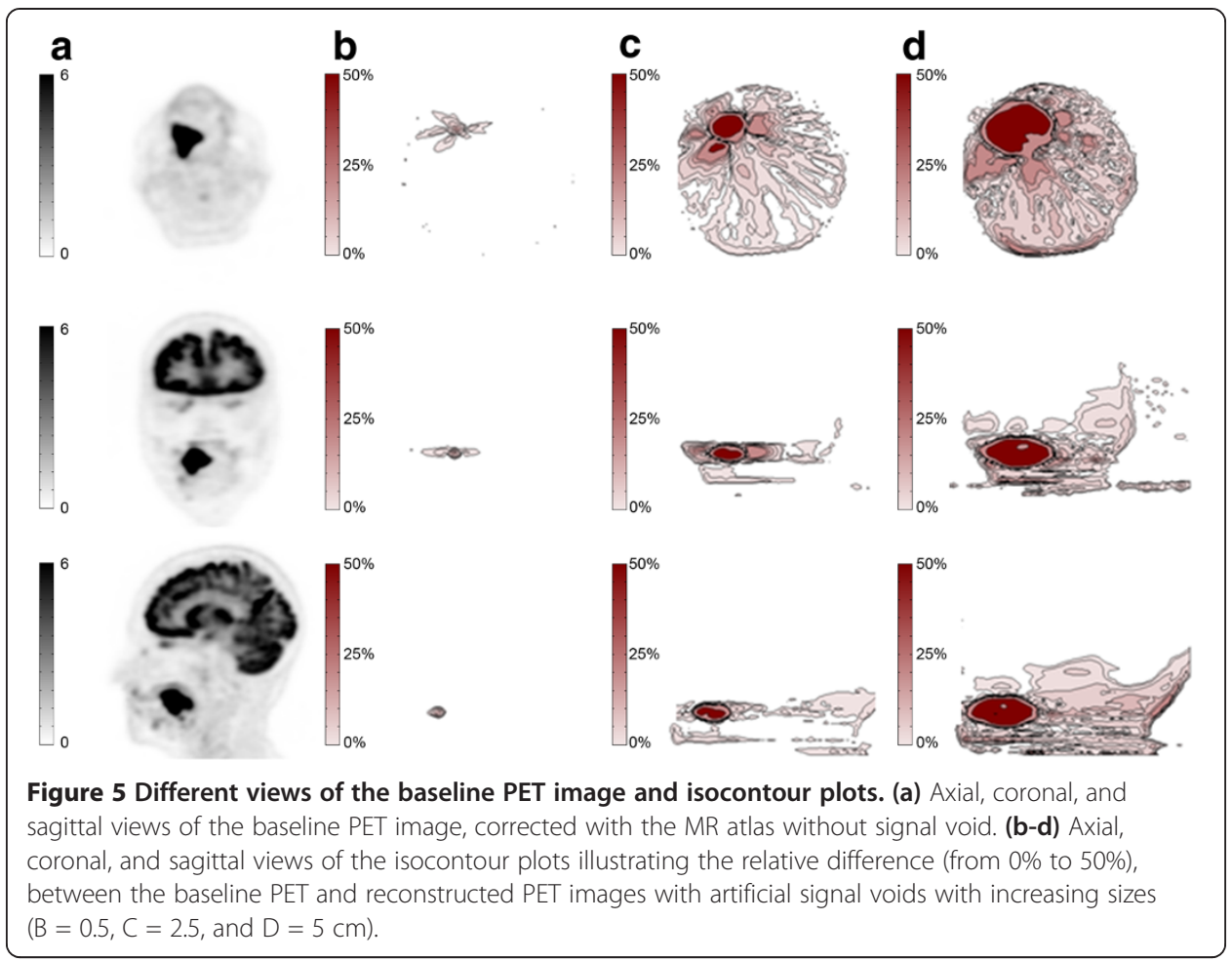

and also will not take into account the true attenuation from metal implants, teeth, or bones.

In addition to a potential improvement of CT-based AC [33], MRI also increases the soft tissue contrast of the oral cavity. This is especially beneficial in FDG PET, since muscle activity can lead to variable FDG uptake in the floor of the mouth [36]. To differentiate this physiological FDG activity from malignancy, it is important that the MRI sequence allows a good anatomical depiction of the oral cavity [17]. T1-FSE images showed better spatial resolution than MAVRIC images in earlier publications [25]. This was also true for the oral cavity in our study with less noise for T1-FSE compared to MAVRIC SL and MAVRIC-fast for most cases. On the other hand, there was a significantly better reduction of spatial blurring for MAVRIC-fast compared to T1-FSE in

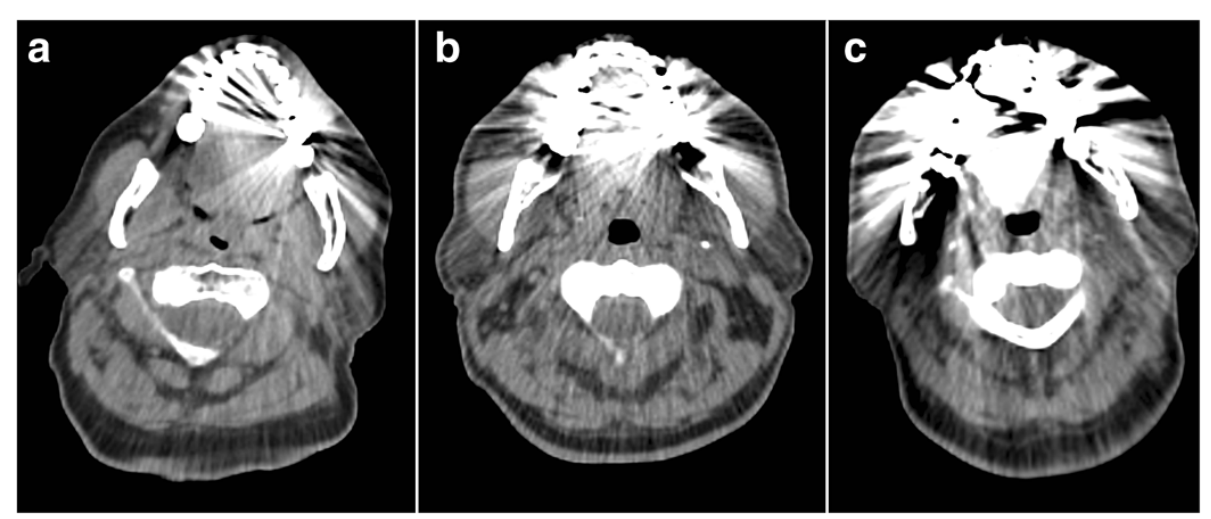

Figure 6 Artifacts by dental implants on the CT images. (a) An axial CT image of the same patient as the MRI images in Figure 3. (b, c) Other patients included in this study. 
patients with large artifacts on LAVA Flex (group 2, Table 3). Regarding image fusion, artifact size did not affect the coregistration between MRI and PET in our trimodality setup, since hardware-based coregistration is independent of image information and visual corrections, which if necessary are performed using bony structures, not affected by focal signal voids.

One limitation of this study is that we could not investigate the alloy composition of the dental implants and had no information of their exact material. Therefore, the influence of different materials on the artifacts in the applied sequences could not be addressed. However, this is the most realistic clinical situation, as patients usually are not aware of the exact composition of their dental implants. A further limitation is a certain uncertainty for measurements that might lead to a slight variability in measured sizes of signal voids and that we only compared vendor-specific sequences and could not investigate SEMAC or compare MAVRIC with SEMAC. Also, volume-selective 3D multispectral imaging (VS-3D-MSI), a fusion of SEMAC and MAVRIC technique, has been reported to provide excellent artifact suppression. In dental imaging, SEMAC reduced artifacts from dental alloys composed of high-susceptibility materials of more than 50\% compared to conventional SE sequences [37]. It might be valuable to have a direct comparison of these two sequences, which have proven their capability of artifact reduction. With the current study, we could not implement MAVRIC-fast for direct AC correction and therefore could not quantify the direct effect on SUV values. With the additional simulation study, we however could show the potential impact of different artifact sizes on SUV values in a real tumor. Future studies with integration of MAVRIC-fast information into the AC map will be needed to further analyze this aspect.

\section{Conclusions}

In summary, this is the first study showing that MAVRIC-fast could be integrated into whole-body PET/MRI imaging for patients with large signal voids due to dental alloys. MAVRIC SL reduced artifact size and increased precise anatomic delineation of the oral cavity. This reduction should improve the accuracy of PET quantification - since large signal voids lead to substantial SUV underestimation. With MAVRIC-fast, acquisition time was kept reasonably short, slightly worsening image noise but further reducing artifact size compared to T1-FSE or MAVRIC SL. Therefore, MAVRIC-fast could be integrated into the diagnostic imaging work-up for head and neck cancer evaluation in patients with dental alloys and potentially also improve AC.

Competing interests

GD is employed by GE Healthcare. All other authors declare that they have no competing interests.

\footnotetext{
Authors' contributions

JMG Collected the data, did a part of the data analysis and drafted the manuscript. GD had the principle study idea, drafted the study design, and edited the manuscript. AB worked on the study design, and drafted the manuscript. MP helped with the MRI protocols and did the data acquisition. HD did further data analysis and edited the manuscript. GKS improved the study design and edited the manuscript. MH was one of the readers and edited the manuscript. PS was one of the readers and edited the manuscript. PV improved the study design and edited the manuscript. IAB did the study design, a part of the data analysis and wrote the manuscript. All authors read and approved the final manuscript.
} 


\section{Author details}

'Department of Medical Radiology, Division of Nuclear Medicine, University Hospital Zurich, Ramistr. 100, CH-8091 Zurich, Switzerland. ${ }^{2}$ Global MR Applications and Workflow, GE Healthcare, Wagistr. 12, CH-8048 Zurich, Switzerland. ${ }^{3}$ Department of Medical Radiology, Institute of Diagnostic and Interventional Radiology, University Hospital Zurich, Ramistr. 100, CH-8091 Zurich, Switzerland. ${ }^{4}$ Department of Medical Radiology, Institute of Neuroradiology, University Hospital Zurich, Ramistr. 100, CH-8091 Zurich, Switzerland.

Received: 19 August 2014 Accepted: 6 November 2014

Published online: 20 December 2014

\section{References}

1. Becker M, Moulin G, Kurt AM, Dulgerov P, Vukanovic S, Zbaren P, Marchal F, Rufenacht DA, Terrier F: Non-squamous cell neoplasms of the larynx: radiologic-pathologic correlation. Radiographics 1998, 18:1189-209. doi:10.1148/ radiographics.18.5.9747615.

2. Loevner LA: Image-guided procedures of the head and neck: the radiologist's arsenal. Otolaryngol Clin North Am 2008, 41:231-50. doi:10.1016/j.otc.2007.10.013.

3. Zbaren P, Becker M, Lang H: Staging of laryngeal cancer: endoscopy, computed tomography and magnetic resonance versus histopathology. Eur Arch Otorhinolaryngol 1997, 254(Suppl 1):S117-22.

4. Ouyang T, Branstetter BF: Advances in head and neck imaging. Oral Maxillofac Surg Clin North Am 2010, 22:107-15. doi:10.1016/..coms.2009.10.002

5. Reske SN, Kotzerke J: FDG-PET for clinical use. Results of the 3rd German Interdisciplinary Consensus Conference, "Onko-PET III", 21 July and 19 September 2000. Eur J Nucl Med 2001, 28:1707-23. doi:10.1007/s002590100626

6. Yamazaki Y, Saitoh M, Notani K, Tei K, Totsuka Y, Takinami S, Kanegae K, Inubushi M, Tamaki N, Kitagawa Y: Assessment of cervical lymph node metastases using FDG-PET in patients with head and neck cancer. Ann Nucl Med 2008, 22:177-84. doi:10.1007/s12149-007-0097-9.

7. Branstetter BF, Blodgett TM, Zimmer LA, Snyderman CH, Johnson JT, Raman S, Meltzer CC: Head and neck malignancy: is PET/CT more accurate than PET or CT alone? Radiology 2005, 235:580-6. doi:10.1148/ radiol.2352040134.

8. Schoder H, Yeung HW, Gonen M, Kraus D, Larson SM: Head and neck cancer: clinical usefulness and accuracy of PET/CT image fusion. Radiology 2004, 231:65-72. doi:10.1148/radiol.2311030271.

9. Huang SH, Chien CY, Lin WC, Fang FM, Wang PW, Lui CC, Huang YC, Hung BT, Tu MC, Chang CC: A comparative study of fused FDG PET/MRI, PET/CT, MRI, and CT imaging for assessing surrounding tissue invasion of advanced buccal squamous cell carcinoma. Clin Nucl Med 2011, 36:518-25. doi:10.1097/RLU.0b013e318217566f.

10. Kuhn FP, Hullner M, Mader CE, Kastrinidis N, Huber GF, von Schulthess GK, Kollias S, Veit-Haibach P: Contrastenhanced PET/MR imaging versus contrast-enhanced PET/CT in head and neck cancer: how much MR information is needed? J Nucl Med 2014, 55:551-8. doi:10.2967/jnumed.113.125443.

11. Moser E, Stadlbauer A, Windischberger C, Quick HH, Ladd ME: Magnetic resonance imaging methodology. Eur J Nucl Med Mol Imaging 2009, 36(Suppl 1):S30-41. doi:10.1007/s00259-008-0938-3.

12. Veit-Haibach P, Kuhn FP, Wiesinger F, Delso G, von Schulthess G: PET-MR imaging using a tri-modality PET/CTMR system with a dedicated shuttle in clinical routine. MAGMA 2013, 26:25-35. doi:10.1007/s10334-012-0344-5.

13. Al-Nabhani KZ, Syed R, Michopoulou S, Alkalbani J, Afaq A, Panagiotidis E, O'Meara C, Groves A, Ell P, Bomanji J: Qualitative and quantitative comparison of PET/CT and PET/MR imaging in clinical practice. J Nucl Med 2014, 55:88-94. doi:10.2967/jnumed.113.123547.

14. Gray CF, Redpath TW, Smith FW, Staff RT: Advanced imaging: magnetic resonance imaging in implant dentistry. Clin Oral Implants Res 2003, 14:18-27.

15. Fellner C, Behr M, Fellner F, Held P, Handel G, Feuerbach S: Artifacts in MR imaging of the temporomandibular joint caused by dental alloys: a phantom model study at T1.5. Rofo 1997, 166:421-428. doi:10.1055/s-2007-1015452.

16. Schenck JF: The role of magnetic susceptibility in magnetic resonance imaging: MRI magnetic compatibility of the first and second kinds. Med Phys 1996, 23:815-850.

17. Eggers $G$, Rieker $M$, Kress B, Fiebach J, Dickhaus $H$, Hassfeld S: Artefacts in magnetic resonance imaging caused by dental material. MAGMA 2005, 18:103-111. doi:10.1007/s10334-005-0101-0.

18. Hubalkova H, La Serna P, Linetskiy I, Dostalova T: Dental alloys and magnetic resonance imaging. Int Dent J 2006, 56:135-141.

19. Petersilge CA, Lewin JS, Duerk JL, Yoo JU, Ghaneyem AJ: Optimizing imaging parameters for MR evaluation of the spine with titanium pedicle screws. AJR Am J Roentgenol 1996, 166:1213-8. doi:10.2214/ajr.166.5.8615272.

20. Suh JS, Jeong EK, Shin KH, Cho JH, Na JB, Kim DH, Han CD: Minimizing artifacts caused by metallic implants at MR imaging: experimental and clinical studies. AJR Am J Roentgenol 1998, 171:1207-13. doi:10.2214/ajr.171.5.9798849.

21. Abbaszadeh K, Heffez LB, Mafee MF: Effect of interference of metallic objects on interpretation of T1-weighted magnetic resonance images in the maxillofacial region. Oral Surg Oral Med Oral Pathol Oral Radiol Endod 2000, 89:759-65.

22. Bezrukov I, Schmidt H, Mantlik F, Schwenzer N, Brendle C, Scholkopf B, Pichler BJ: MR-based attenuation correction methods for improved PET quantification in lesions within bone and susceptibility artifact regions. J Nucl Med 2013, 54:1768-1774. doi:10.2967/jnumed.112.113209.

23. Tormanen J, Tervonen O, Koivula A, Junila J, Suramo I: Image technique optimization in MR imaging of a titanium alloy joint prosthesis. J Magn Reson Imaging 1996, 6:805-811.

24. Chen CA, Chen W, Goodman SB, Hargreaves BA, Koch KM, Lu W, Brau AC, Draper CE, Delp SL, Gold GE: New MR imaging methods for metallic implants in the knee: artifact correction and clinical impact. J Magn Reson Imaging 2011, 33:1121-1127. doi:10.1002/jmri.22534. 
25. Hayter CL, Koff MF, Shah P, Koch KM, Miller TT, Potter HG: MRI after arthroplasty: comparison of MAVRIC and conventional fast spin-echo techniques. AJR Am J Roentgenol 2011, 197:W405-11. doi:10.2214/AJR.11.6659.

26. Sutter R, Hodek R, Fucentese SF, Nittka M, Pfirrmann CW: Total knee arthroplasty MRI featuring slice-encoding for metal artifact correction: reduction of artifacts for STIR and proton density-weighted sequences. AJR Am J Roentgenol 2013, 201:1315-1324. doi:10.2214/AJR.13.10531.

27. Sutter R, Ulbrich EJ, Jellus V, Nittka M, Pfirrmann CW: Reduction of metal artifacts in patients with total hip arthroplasty with slice-encoding metal artifact correction and view-angle tilting MR imaging. Radiology 2012, 265:204-214. doi:10.1148/radiol.12112408.

28. Koch KM, Brau AC, Chen W, Gold GE, Hargreaves BA, Koff M, McKinnon GC, Potter HG, King KF: Imaging near metal with a MAVRIC-SEMAC hybrid. Magn Reson Med 2011, 65:71-82. do:10.1002/mrm.22523.

29. Samarin A, Burger C, Wollenweber SD, Crook DW, Burger IA, Schmid DT, von Schulthess GK, Kuhn FP: PET/MR imaging of bone lesions - implications for PET quantification from imperfect attenuation correction. Eur J Nucl Med Mol Imaging 2012, 39:1154-1160. doi:10.1007/s00259-012-2113-0.

30. Drzezga A, Souvatzoglou M, Eiber M, Beer AJ, Furst S, Martinez-Moller A, Nekolla SG, Ziegler S, Ganter C, Rummeny EJ, Schwaiger M: First clinical experience with integrated whole-body PET/MR: comparison to PET/CT in patients with oncologic diagnoses. J Nucl Med 2012, 53:845-855. doi:10.2967/jnumed.111.098608.

31. Landis JR, Koch GG: An application of hierarchical kappa-type statistics in the assessment of majority agreement among multiple observers. Biometrics 1977, 33:363-374.

32. Neimatallah MA, Chenevert TL, Carlos RC, Londy FJ, Dong Q, Prince MR, et al: Subclavian MR arteriography: reduction of susceptibility artifact with short echo time and dilute gadopentetate dimeglumine. Radiology 2000, 217:581-586. doi:10.1148/radiology.217.2.r00oc37581.

33. Delso G, Wollenweber S, Lonn A, Wiesinger F, Veit-Haibach P: MR-driven metal artifact reduction in PET/CT. Phys Med Biol 2013, 58:2267-2280. doi:10.1088/0031-9155/58/7/2267.

34. Buchbender C, Hartung-Knemeyer V, Forsting M, Antoch G, Heusner TA: Positron emission tomography (PET) attenuation correction artefacts in PET/CT and PET/MRI. Br J Radiol 2013, 86:20120570. doi:10.1259/bjr.20120570.

35. Keller SH, Holm S, Hansen AE, Sattler B, Andersen F, Klausen TL, Højgaard L, Kjær A, Beyer T: Image artifacts from MR-based attenuation correction in clinical, whole-body PET/MRI. MAGMA 2013, 26:173-181. doi:10.1007/ s10334-012-0345-4.

36. Haerle SK, Hany TF, Ahmad N, Burger I, Huber GF, Schmid DT: Physiologic [18F]fluorodeoxyglucose uptake of floor of mouth muscles in PET/CT imaging: a problem of body position during FDG uptake? Cancer Imaging 2013, 13:1-7. doi:10.1102/1470-7330.2013.0001.

37. Zho SY, Kim MO, Lee KW, Kim DH: Artifact reduction from metallic dental materials in T1-weighted spin-echo imaging at 3.0 tesla. J Magn Reson Imaging 2013, 37:471-8. doi:10.1002/jmri.23785.

doi:10.1186/s40658-014-0102-z

Cite this article as: Gunzinger et al:: Metal artifact reduction in patients with dental implants using multispectral three-dimensional data acquisition for hybrid PET/MRI. EJNMMI Physics 2014 1:102.

\section{Submit your manuscript to a SpringerOpen ${ }^{\circ}$ journal and benefit from:}

- Convenient online submission

- Rigorous peer review

- Immediate publication on acceptance

- Open access: articles freely available online

- High visibility within the field

- Retaining the copyright to your article 